\title{
Polyelectrolytes in Multivalent Salt Solutions: Monomolecular versus Multimolecular Aggregation
}

\section{Ching-I Huang*,† and Monica Olvera de la Cruz}

Materials Science and Technol ogy Program, Graduate Institute of Engineering Technol ogy, National Taiwan University of Science and Technology, Taipei 106, Taiwan, and Department of Materials Science and Engineering, Northwestern University, Evanston, Illinois 60208

Received April 26, 2001; Revised Manuscript Received November 2, 2001

\begin{abstract}
We analyze the segregation of strongly charged chains of $\mathrm{N}$ monomers of size $a$ in the presence of multivalent salts with valence $z$ as a function of the concentration of monomers $\phi$ in the solutions. The multivalent ions of opposite charge condense along the monomers and induce monomer attractions that lead to the formation of dense finite size aggregates (micelles) of chains and multivalent ions when their valence $z>1$. We compute the number density of chains in aggregates with $p=1,2,3$, $4, \ldots$ chains by equating the chemical potential of all the $p$-aggregated chains and of the free and condensed ions in the aggregates. At low concentration of multivalent salts $m$, we observe monomolecular $(p=1)$ preci pitated chains when $\phi<\phi^{*}$, where $\phi^{*} \cong \mathrm{mz}$. When $\phi$ increases above $\phi^{*}$, the chains redissolve in the solution and adopt stretched conformations. As m increases above a critical value $\mathrm{m}^{* *}$, the number density of aggregates with $p>1$ chains increases such that there are more aggregates with $p^{*}>1$ chains when $\phi^{* *}<\phi<\phi^{*}$. If we only include the surface free energy in the analysis, we find $\mathrm{p}^{*}=\infty$. However, if we include the chain entropy of confining $p$ chains in a region $R(p)=(p N)^{1 / 3} a$ in the free energy, $p^{*}$ can be finite and greater than or equal to one. This situation arises when the chains are constrained to stretched conformations, such as in metastable toroidal and in spherical coil aggregates observed in long doublestranded DNA in multivalent ions.
\end{abstract}

\section{Introduction}

Linear polyelectrolytes are typically water-soluble at low ionic strength monovalent salt solutions due to a net repulsion between the charged monomers. In the presence of multivalent ions or molecules of high valence of opposite charge, however, the chains precipitate into highly compact structures. These precipitates have been observed experimentally ${ }^{1-5}$ and by computer simulations. ${ }^{6-11}$ The electrostatic attractions due to the condensed multivalent ions are responsible for the preci pitation of synthetic polyelectrolytes, such as poly(styrenesulfonate) in the presence of $\mathrm{La}^{3+}$ or $\mathrm{Th}^{4+}$ ions ${ }^{12}$ and of poly(acrylic acid)s in the presence of divalent and higher valence salts ${ }^{5}$ as well as for the precipitation of DNA in the presence of polyamines ${ }^{4,13}$ or of cobalt hexamine. ${ }^{1}$

Counterion-mediated attractions have been predicted by various models. $8,9,12,14-23$ I on bridging type models which assume a local interaction between the monomers via condensed multivalent ions al ong the chains have been extensively used to explain the multivalentinduced precipitation of chains.4,12,15,19 Gonzalez-M ozuel os and Olvera de la $\mathrm{Cruz}^{14}$ showed that nonlocal type interactions due to electrostatic correlations among condensed counterions and monomers also lead to the precipitation of the chains when the valence of the counterions $z$ is sufficiently large and/or when the charge density along the chains is very high. They showed that these correlations collapse the chains into a dense state. In flexible chains a sphere of monomers neutralized by the hydrated counterions is expected, and

\footnotetext{
† National Taiwan University of Science and Technology.

‡ Northwestern University.

* Author for correspondence. E-mail chingi@mail.ntust.edu.tw; Fax 886-2-27376429.
}

in semiflexible chains, such as double-stranded DNA, the dense state is a toroid or a spherical coil depending on the length of the DNA (very long chains form spherical coil aggregates). ${ }^{24}$ Many liquid theories, ${ }^{12-16,19,20}$ which include correlations in Poisson-Boltzmann meanfiel d type approaches, ${ }^{25,26}$ al so predict the precipitation of the chains. However, liquid theories, either virial models that include short-range correlations or DebyeHuckel type models that include the contribution from charge fluctuations, though reasonable for dilute solutions in monovalent ions, ${ }^{27}$ cannot be used to describe the resulting dense precipitate of positive and negative charges observed in the presence of multivalent ions. Since the cohesive energy in a neutral precipitate of charges is much larger than the thermal energy $k_{B} T$ when the ions are multivalent, the cohesive energy is computed by using solid-state physics models, as first suggested by Rouzina and Bloomfield. ${ }^{17}$ The cohesive energy of an ionic solid is proportional to the Coulomb energy of the neutral minimum cluster (unit cell).28 Therefore, it is inversely proportional to the distance between charges, which is determined by the size of the multivalent counterions and the charge density along the polyelectrolytes. ${ }^{17}$ I ndeed, the precipitation induced by counterion-mediated attractions is mostly effective by small counterions, ${ }^{29}$ in agreement with the models that lead to strongly correlated multivalent ions between two aligned charged plates ${ }^{17,30}$ and between two aligned rods. ${ }^{18}$ The dense state in flexible and semiflexible chains resembles an ionic glass structure due to the disorder.10,11,21,22

In chains of long degree of polymerization $\mathrm{N}$ with each monomer carrying a charge, the precipitation of dilute polyelectrolytes in multivalent salt solutions has been studied using a two-state model. ${ }^{21}$ One state consists of a stretched (rodlike or coillike depending on the 
concentration of monovalent salts) conformation and the other of a dense precipitate with one chain. As discussed above, the dense conformation does not have freely fluctuating ions, and therefore the electrostatic interactions inside the precipitate are not screened in the Debye-Huckel screening sense. Instead, the cohesive energy of the preci pitate is estimated assuming it is an ionic solid of a finite radius. ${ }^{21}$ That is, it is proportional to the Coulomb electrostatic energy (monopol e term) of a minimum neutral cluster of z monomers compensated by a counterion of valence $z$ (the minimization yields nearly zero monovalent ions inside the precipitate because they have more entropy outsi de the aggregates where they do contribute to screening and have less cohesive energy in the precipitated state than the multivalent ions). ${ }^{21}$ In ionic crystals the electrostatic interactions with further neighbors are absorbed in the proportionality constant in the cohesive energy, the Madelung constant, which is of the order of one in monoval ent ionic solids. ${ }^{31}$ If the amount of monovalent salt present in the system is low, the transition predicted with this two-state model occurs when the ratio of polyelectrolyte concentration to multivalent salt concentration is about $z,{ }^{21}$ in agreement with the experimental results. ${ }^{4}$

The two-state model gives upon minimization aggregates of precipitated chains with $p^{*}=1$ or $p^{*}=\infty$, where $p^{*}$ is the most probable number of chains per aggregate, depending on the value of the surface energy when the entropy of the chains is neglected. ${ }^{21} \mathrm{~F}$ or good solvent conditions the precipitates are monomol ecular, and for poor solvent conditions they are infinite aggregates. If only the electrostatic surface energy is included, the situation resembles a poor solvent condition where macroscopic segregation is expected regardless of the specific model used for the counterion-mediated attractions. 22,32 However, monomolecular and multimolecular aggregates of finite size have been observed in doublestranded (semiflexible) long DNA dilute solutions in the presence of multivalent salts. 1,3,4,24,33,34 Finite size bundles have also been observed in short DNA fragments, ${ }^{4,33}$ which are rigid rods. In rigid rods, both analysis ${ }^{35}$ and modeling ${ }^{36}$ suggest that the bundles do not grow to an infinite aggregate size due to kinetic effects.

In this paper we address the possibility of metastable polydisperse aggregates of charged chains and condensed ions by extending the two-state model to a thermodynamic model that allows for a distribution of clusters of various size $p=1,2,3,4, \ldots$; that is, the precipitated system consists of dense aggregates of nearly neutralized chains by condensed counterions that have different number of aggregated chains $p=1,2,3$, $4, \ldots$. The number density of aggregates with $p$ chains per aggregate $\left(n_{p}\right)$ is obtained by equating the chemical potential of the chains in the different aggregates and the chemical potential of the condensed ions in the aggregates and the free counterions. We compute the free energy of the aggregates with $p=1,2,3,4, \ldots$ chains by using the ionic solid model described above. Therefore, the aggregated chains do not obey random walk statistics as expected in melts with only van der Waals interactions, because the cohesive energy per monomer in the aggregates of monomers and multivalent ions is much larger than $k_{B} T$. We find that the entropy reduction of the chains aggregated into dense micelles is essential to find polydisperse aggregates of intermediate sizes. Though this polydisperse state of aggregates may not be the lowest energy state of the system, the disorder due to chain flexibility and entanglements can lead to local (not global) equilibrium conformations that may be observed experimentally. Similar kinetic effects are observed in crystallization processes in long multichain systems. Though the lowest energy state is a macroscopic crystal of highly elongated chains, micelles or crystalline clusters of about $10 \mathrm{~nm}$ form. Here we also expect a perfect ionic crystal neutralized completely by the counterions to have lowest energy. However, kinetics and disorder make this possi bility physically impossible to observe experimentally. Motivated by this fact, we explore the possibility that an entropic reason leads to the observation of finite size aggregates.

Borue and Erukhimovich ${ }^{37}$ discussed the possibility of segregation at intermediate length scales for flexible weakly charged polyelectrolytes at low ionic strength solutions in a bad solvent by using the random phase approximation (RPA). RPA is a liquid state theory for macromolecules equivalent to the Debye-Huckel theory; that is, charge fluctuations are included in the free energy functional by linearizing the correlation and including only long-range electrostatic interactions. They predicted a microphase structure due to the competition of the bad solvent effects that lead to macroscopic phase separation and the entropy of mixing of the counterions that opposes strongly macroscopic phase separation of the long polyelectrolytes and the bad solvent. Multivalent condensed counterions act as an extremely bad solvent to the chains given that they generate effective attractions between the charged monomers. Instead of a microphase structure, however, we expect finite size aggregates of strongly perturbed chains given that the attractions mediated by the multivalent ions are much larger than the thermal energy $k_{B} T$, invalidating the use of RPA, which assumes Gaussian chain statistics. These attractions lead to strong short-range correlations, which have been ignored in RPA. In this paper we implement the model of strongly correlated finite size condensed ions ${ }^{17,18,21,22}$ in a micelle approach of charged systems ${ }^{39,40}$ incorporating highly perturbed conformations.

It is known that double-stranded DNA wraps around itself circumferentially in the presence of multivalent particles and that in many cases only few chains wrap around. Nonelectrostatic mean-field type methods have been used to study the monomolecular precipitation of these semiflexible chains into toroids. ${ }^{24,41}$ The toroids, however, are only observed at intermi diate semiflexible chain sizes. When the chain is very long, the precipitation of semiflexible molecules has been observed and predicted to be into a rather spherical coil. ${ }^{24}$ Vasilevskaya et al. ${ }^{24}$ use simple scaling forms to compute the entropy reduction in the spherical monomolecular precipitates of chains, such as the standard entropy decrease of stretched chains ${ }^{42}$ and of collapsed chains. ${ }^{43}$ We follow a similar approach, but we include many chain aggregates, hereafter referred to as micelles. A micelle approach to study many chain multivalentinduced aggregation ignoring el ectrostatic effects was first proposed by Park et al. ${ }^{34}$ They only analyze monodispersed toroids and conclude that finite size toroids are observed rather than an infinite phase of precipitated DNA due to topological defects encounter as the toroid grows by wrapping around more chains. We expl ore here the case of very long chains where the 
toroid is very large and resembles a spherical aggregate. In the limit of long chains we can use the standard entropy reduction of flexible chains to describe the entropy reductions, following Vasilevskaya et al.24 Notice that there are two possible scenarios for the entropy change of confining $p$ chains each with $\mathrm{N}$ monomers of length a into a dense aggregate of size $R(p)=(p N)^{1 / 3} a$. Either the chains form macroscopic aggregates $\left(p^{*}=\infty\right)$ of monomolecular precipitated chains of radius $\mathrm{N}^{1 / 3} \mathrm{a}$, or they form spherical aggregates of "interpenetrated" chains of a size $R(p)=(p N)^{1 / 3} a$. We show here that though the first possibility may have lower energy in flexible unconstrained chains, there is a metastable state of multimolecular interpenetrated stretched chains in aggregates of size $\mathrm{p}^{*} \sim$ Nz that may be observed experimentally due to kinetics effects associated with forming an infinite cluster of chains. This entropic effect may explain the finite size toroidal bundles observed in semiflexible chains in multivalent salt solutions.1,33,34 When the radius of the toroid increases by adding more chains to the bundle, the entropy reduction increases, possibly giving rise to a finite value of $p^{*}(\mathrm{~N})$.

In section II we describe the theoretical approach for flexible chains and the equations used for the different terms in the free energy functional. As explained above, we use standard entropy reductions in the preci pitated ${ }^{43}$ $(p=1,2,3, \ldots)$ and stretched ${ }^{42}(p=1)$ states and the solid-state physics approach for the el ectrostatic contributions. ${ }^{21,22}$ We explain the differences between unconstrained chains and chains constrained to stretched conformations. For simplicity, we do not consider the possibility of necklace conformations expected between the stretched and collapsed states in bad solvent conditions. ${ }^{44-47}$ Though this simplification may lead to errors in the transition from stretched to collapsed in flexible chains, in semiflexible chains, where finite size aggregates are predicted, it is more difficult to form necklaces between the stretched and spherical precipitated states. In section III we describe the results for mutivalent salts of valence $z=2$ and 3 , and in section IV we give the conclusions.

\section{Theoretical Approach}

In this section we construct the free energy of monodisperse polyelectrolyte chains in the presence of a solvent at temperature T with a bulk dielectric constant $\epsilon$. We assume that there are two possible states for the chains: stretched and precipitated. In addition, we consider the aggregation of precipitated chains in the presence of monovalent and multivalent salt solutions. In section II.a, we calculate the free energy of precipitated polyelectrolytes aggregating into micelles with $p$ chains per aggregate, $p=1,2,3, \ldots$. In section II .b we calculate the free energy of extended single chains. By comparing the free energy of each possible state, we determine the conditions under which the stretched single chains, the collapsed single chains, or the multiple aggregated micelles are preferred.

II.a. Monomolecular and Multimolecular Aggregation of Polyelectrolyte Chains. We assume that each chain has $\mathrm{N}$ monomers, and each monomer is positively charged with valence equal to 1 . The solution contains monovalent salts with concentration $\mathrm{s}$ and multivalent salts with concentration $\mathrm{m}$ and valence $z$. The total monomer concentration of polyelec- trolytes is $\phi$, and the concentration of polyelectrolytes aggregated with $p$ chains is $\phi_{p}$, such that

$$
\phi=\sum_{\mathrm{p}=1,2, \ldots} \phi_{\mathrm{p}}
$$

Therefore, the number density of aggregates with $p$ chains per aggregate, $n_{p}$, is then equal to $\phi_{p} /(p N)$. Initially, the concentration of negative monoval ent and multivalent counterions is $\mathrm{s}+\phi$ and $\mathrm{m}$, respectively. Since the fraction of monovalent and multivalent counterions condensed in a given size aggregates depends on the size of the aggregate, we denote $f_{s}(p)$ and $f_{m}(p)$ the fraction of monomer charge in every $p$-aggregated chain compensated by the condensed monovalent and multivalent counterions, respectively. We then have $f_{s}(p) N$ and $f_{m}(p) N / z$ condensed negative monovalent and multivalent ions per chain, where $f_{s}(p)$ and $f_{m}(p)$ are determined self-consistently by the minimization process. Therefore, the concentration of free negative monovalent ions is

$$
\mathrm{n}_{\mathrm{s}}^{\mathrm{f}}=\mathrm{s}+\phi-\sum_{\mathrm{p}=1,2, \ldots} \mathrm{n}_{\mathrm{p}} \mathrm{pNf}_{\mathrm{s}}(\mathrm{p})
$$

The concentration of free negative multivalent ions is equal to

$$
\mathrm{n}_{\mathrm{m}}^{\mathrm{f}}=\mathrm{m}-\sum_{\mathrm{p}=1,2, \ldots} \frac{\mathrm{n}_{\mathrm{p}} \mathrm{pNf} \mathrm{f}_{\mathrm{m}}(\mathrm{p})}{\mathrm{z}}
$$

The concentration of free positive monovalent and multival ent counterion is simply s and $\mathrm{m}$, respectively, since none of them condense to the polyelectrolyte chains.

Each p-aggregated micelle in a precipitated state of $p$ chains is contained within a sphere of average radius $R(p)$, such that

$$
R^{3}(p)=a^{3} p N\left(1+f_{s}(p)+f_{m}(p) / z\right)
$$

where $a$ is the size of each monomer. That is, the water is ignored because we assume that the aggregates are dense systems of monomers and condensed hydrated monovalent and multivalent ions with a hydrated size of the order of the monomer size a.

We assume that the concentration of aggregates is very low, so that the average distance between the aggregates is much larger than the maximum value of $R(p)$. Furthermore, since after the minimization the effective charge of the aggregates is strongly reduced by the condensed counterions, we neglect the interactions among the aggregates. Indeed, it has been demonstrated that the electrostatic interaction between nearly neutralized charged spheres by condensed counterions is very short range. ${ }^{48,49}$ The multipole fluctuations and polarizability of charge induced by the nearby neutralized colloids are short-range (of the order of the size of the ions) and negligible.49 Therefore, we neglect all the electrostatic interactions in dilute concentrations of nearly neutral aggregates.

The free energy per unit volume of the system is given by

$$
F N=F_{0} N+F_{C} N
$$

where $\mathrm{V}$ is the total vol ume, $\mathrm{F}_{0}$ is the free energy of the system when monomer is uncharged, and $F_{c}$ is the 
contribution to the free energy due to the charge of the monomers and the free counterions. By using the simplest mean-field theory,

$$
F_{0} N=F_{t p} N+\sum_{p} n_{p} F_{i d}(p)
$$

where

$$
\mathrm{F}_{\mathrm{tp}} \mathrm{N}=\mathrm{k}_{\mathrm{B}} \mathrm{T} \sum_{\mathrm{p}} \mathrm{n}_{\mathrm{p}} \ln \phi_{\mathrm{p}} \mathrm{a}^{3}
$$

is the translational entropy of the aggregates, and $F_{i d}(p)$ is the free energy reduction of $p$ chains confined to $R(p)$, given by 24,43

$$
F_{i d}(p)=k_{B} T p\left(\frac{3}{2} \frac{R^{2}(p)}{N a^{2}}+\frac{\pi^{2}}{6} \frac{N a^{2}}{R^{2}(p)}-3.1449\right)
$$

On the right-hand side of eq 8 , we add the two limits of confined random walks of unperturbed chains of size $R_{0}=N^{1 / 2}$ a to a region of size $R(p)$, where $R(p)$ is given by eq 4; the first term is for $R_{0} \gg R(p)$, the second one is for $R_{0} \ll R(p)$, and the last term is to ensure that the change in free energy is zero when $R_{0}=R(p)$. The first term on the right-hand side of eq 8 is not applicable to describe collapsed chains given that in the collapsed state the chains are not constrained to be expanded by any force. (The electrostatic force that stretches the chains when the concentration of multivalent particles is low is not present in the collapsed or aggregated state because this state is nearly neutral.) However, in double-stranded DNA and in other semiflexible polyelectrolytes, it is well-known that a monomolecular collapse occurs first to a toroidal conformation when $\mathrm{N}$ is sufficiently large. In that case, the initial toroid acts like a seed to other chains to add to the cluster. The cluster stops growing after certain size is reached because the entropy of the chains increases due to a stretching of the chains to the size of the toroid. The volume occupied by the chains in the toroid is the circumference of the toroid equal to $2 \pi \mathrm{R}$ multiplied by the area of the toroid equal to $\pi r^{2}$. When $\mathrm{N}$ is large and the toroid grows, $r$ is of the order of $R$, and the volume occupied by the toroid is given by $2 \pi^{2} \mathrm{R}^{3} \sim \mathrm{pN}$, leading for large $p$ and $N$ to $R(p) \sim(p N)^{1 / 3}$ a. Therefore, when $N$ is large (when the hole in the toroid center is negligible), eq 8 does describe the stretching of the chains when they add to the toroid. The first term in eq 8, applicable to these constraint chains when $\mathrm{R}>\mathrm{N}^{1 / 2} \mathrm{a}$, is responsible for a finite most probable micelle size $\mathrm{p}^{*}$ in the aggregated state. In eqs 34 and 35 in section III we find by scaling that the most probable value of the number of chains aggregated in the micelles is indeed determined by the balance of the surface energy term (derived in eq 16) and the first term on the right-hand side of eq 8 . Notice that with eq 8 we are only describing a metastable state. The lowest energy state will give an aggregated size $p^{*}=\infty$ for both flexible and semiflexible chains. Since this is a macroscopic phase, a phase diagram has to be computed. However, this macroscopic phase corresponding to $\mathrm{p}^{*}=\infty$ is impossible to observe in semiflexible chains due to kinetic effects. In flexible unconstrained chains, however, the aggregated chains are collapsed due to the strong short-range electrostatic attractions generated by the multivalent ions, and even if they are initially in a monomolecular collapsed state (obtained setting $R(p=1)$ in the second term on the right-hand side of eq 8) or if they form a homogeneous aggregate of collapsed chains with $R<N^{1 / 2} a$, their entropy per micelle with $p$ chains decreases as $p$ increases favoring an infinite aggregate.

The el ectrostatic contribution to the free energy in eq $5, F_{c}$, contains the translational entropy of the free counterions $\mathrm{F}_{\mathrm{t}}$, the electrostatic interactions among all of the free ions $F_{e}$, the internal excess free energy of the net polyion due to the Coulombic interactions $F_{e s}$, the el ectrostatic attraction free energy of the bulk in the precipitated state $F_{e b}$, and the electrostatic contribution to the interfacial free energy for finite size $R(p)$ of $p$-aggregates $F_{\text {ein }}(p)$ :

$$
\mathrm{F}_{\mathrm{C}} \mathrm{N}=\mathrm{F}_{\mathrm{ti}} \mathrm{N}+\mathrm{F}_{\mathrm{e} i} \mathrm{~N}+\mathrm{F}_{\mathrm{es}} \mathrm{N}+\mathrm{F}_{\mathrm{eb}} \mathrm{N}+\mathrm{F}_{\mathrm{ein}} \mathrm{N}
$$

We use an ideal translational energy form,

$$
\begin{aligned}
& \mathrm{F}_{\mathrm{ti}} N=\mathrm{k}_{\mathrm{B}} \mathrm{T}\left(\mathrm{s} \ln s \mathrm{a}^{3}+\right. \mathrm{m} \ln \mathrm{ma} \mathrm{a}^{3}+ \\
&\left.\mathrm{n}_{\mathrm{s}}^{\mathrm{f}} \ln \mathrm{n}_{\mathrm{s}}^{\mathrm{f}} \mathrm{a}^{3}+\mathrm{n}_{\mathrm{m}}^{\mathrm{f}} \ln \mathrm{n}_{\mathrm{m}}^{\mathrm{f}} \mathrm{a}^{3}\right)
\end{aligned}
$$

The electrostatic interactions among all of the free ions $\mathrm{F}_{\mathrm{e}}$ are expressed in the Debye-Huckel form,

$$
\mathrm{F}_{\mathrm{e}} N=-\frac{\mathrm{k}_{\mathrm{B}} T \kappa_{\mathrm{f}}^{3}}{12 \pi}
$$

where $\kappa_{\mathrm{f}}^{-1}$ is the net Debye length determined by

$$
\kappa f^{2}=\left.4 \pi\right|_{B}\left(s+m z^{2}+n_{s}^{f}+n_{m}^{f} z^{2}\right)
$$

where $I_{B} \equiv \mathrm{e}^{2} /\left(k_{B} T \epsilon\right)$ is the Bjerrum length. The DebyeHuckel approximation for point ions, eq 11, is only valid at very dilute concentration of ions when the solution is electrically neutral.50 In the precipitated state the aggregates are nearly neutral. However, in the stretched conformation there is an excess of counterions noncondensed al ong the chains. In this case one should use a neutral cell model, ${ }^{26}$ where each cell contains one polyelectrolyte and its counterions. The neutral cell model for spherical polyelectrolyte solutions at low salt concentrations does recover Debye-Huckel screening laws for the counterions far away from the polyelectroIytes in dilute polyel ectrolyte solutions. It al so gives an effective reduced charge for the polyel ectrolytes by the condensed counterions. As pointed out by Alexander et al.,26 the effective charge of the polyelectrolytes can be computed assuming that condensed ions reduce the monopole electrostatic energy term. The fraction of condensed counterions is determined self-consistently by equating the chemical potential of the condensed and free counterions. We follow here a similar approximation to determine the effective charge of the aggregates (see eq 13) and for the stretched conformation (see eq 29). Also, we include the fluctuations of the free counterions using the Debye-Huckel law, which they showed is valid when the solution is sufficiently dilute in salts and in polyelectrolytes. When the concentration of ions increases, one has to use the full Debey-Huckel approximation, ${ }^{51}$ the modified mean spherical approxi- 
mation, 52 or other models that include the size of the ions ${ }^{53}$ to avoid spurious results predicted by DebyeHuckel models of points ions, such as phase separation of polyel ectrolytes semidilute solutions in monovalent salts. ${ }^{54} \mathrm{~A}$ big difference between our approach and the nonlinear Poisson-Boltzmann approach of Alexander et al. ${ }^{26}$ and of others is the inclusion of strong correlations in the free energy (see eq 15).

The monopole term in the excess free energy of the net polyion due to the Coulombic interactions $F_{\text {es }}$ has the form

$$
F_{\text {es }} N=k_{B} T \sum_{p} n_{p} \frac{\mathrm{I}_{B}}{R(p)} Z_{\text {eff }}^{2}
$$

where the net charge of a $p$-aggregate $Z_{\text {eff }}$ is equal to

$$
Z_{\text {eff }}=p N\left(1-f_{s}(p)-f_{m}(p)\right)
$$

The electrostatic attraction energy of the bulk for $p$-aggregated chains in the precipitated state $F_{e b}$ is given by ${ }^{21}$

$F_{e b} N=-1.71 k_{B} T \frac{I_{B}}{a} \sum_{p} n_{p} p N\left(f_{s}(p)+\frac{f_{m}(p)(z+1)}{2}\right)$

In eq 15 we use the model of an ionic crystal with a cohesive energy computed as follows. We have assumed that all small distances in the system are of order a. We cluster one counterion with all the monomers that are required to compensate its charge, 1 for monovalent, and $z$ for multivalent ions. If all the particles in the cluster compensated by ions of valence $z$ are at about the same distance from each other, the Coulombic energy contribution of the cluster is $E /\left(k_{B} T\right)=-I_{B} z(z+1) /$ (2a). This is the first term in the series for the effective Madel ung constant. There are $\mathrm{pf}_{\mathrm{s}} \mathrm{N}$ clusters with monovalent counterions and $\mathrm{pf}_{\mathrm{m}} \mathrm{N} / \mathrm{z}$ with multivalent counterions. The proportional ity constant in the monopole term used in eq 15, the Madelung constant, was obtained numerically for a neutral spherical aggregate of radius $\mathrm{R}$ in a three-dimensional Cartesian coordinate space ${ }^{55}$ and is added in eq 15 to recover the results for monovalent ionic solids in a cubic lattice. For each element the interaction between it and every other element in the sphere is summed. For monovalent finite size salt crystals, the total free energy divided by the number of positive atoms that contributed to the free energy plotted vs the radius $\mathrm{R}$ shows a convergence from above to -1.71 for large values of $R$. The correction for small values of $R$ can be fit to $0.5 R^{-1}$. This is a deviation due to the surface energy of our finite aggregates. Therefore, if one modifies the coefficient to include the multivalent ion clusters, the electrostatic surface free energy for finite size $R(p)$ of $p$-aggregates $F_{\text {ein }}(p)$ has the form

$F_{\text {ein }} N=0.5 k_{B} T I_{B} \sum_{p} \frac{n_{p} p N}{R(p)}\left(f_{s}(p)+\frac{f_{m}(p)(z+1)}{2}\right)$

which is often rewritten as

$$
\mathrm{F}_{\text {ein }} N=\mathrm{k}_{\mathrm{B}} T \sum_{\mathrm{p}} \mathrm{n}_{\mathrm{p}} 4 \pi \mathrm{R}^{2}(\mathrm{p}) \gamma
$$

where $\gamma$ is the surface energy per unit area. In poor solvent conditions $\gamma$ has an extra thermodynamic contribution besides the electrostatic contribution determined from eqs 4, 16a, and 16b. Here we assume good solvent conditions.

Once the free energy is constructed, we minimize it by equating (i) the chemical potential of the chains in all of the $p$-aggregates with $p=2,3, \ldots$ and the chemical potential of the free chains $(p=1)$, i.e.,

$$
\mu_{\mathrm{p}}=\mathrm{p} \mu_{1} \quad \mathrm{p}=2,3, \ldots
$$

where $\mu_{\mathrm{p}}=\partial(\mathrm{F} N) / \partial \mathrm{n}_{\mathrm{p}}$. This condition is often rewritten as

$$
\ln \phi_{\mathrm{p}}=\mathrm{p} \ln \phi_{1}-\left(\mu_{\mathrm{p}}^{\circ}-\mathrm{p} \mu_{1}^{\circ}\right)
$$

where $\mu_{\mathrm{p}}^{\circ}$ is the p-aggregate standard pseudochemical potential, (ii) the chemical potential of the free monovalent counterions and the chemical potential of the monovalent counterions condensed to the $p$-aggregates for $p=2,3, \ldots$,

$$
\frac{\partial(\mathrm{F} N)}{\partial \mathrm{n}_{\mathrm{s}}^{f}}=\frac{\partial(\mathrm{F} N)}{\partial\left(\mathrm{n}_{\mathrm{p}} \mathrm{pNf}_{\mathrm{s}}\right)}
$$

and (iii) the chemical potential of the free multivalent counterions and the chemical potential of the condensed multivalent ions to the $p$-aggregates for $p=2,3, \ldots$,

$$
\frac{\partial(F / N)}{\partial n_{m}^{f}}=\frac{\partial(F /)}{\partial\left(n_{p} p N f_{m} / z\right)}
$$

The numerical procedure is described as follows. First of all, we input the initial conditions, such as $\mathrm{s}, \mathrm{m}, \phi$, $\mathrm{n}_{\mathrm{s}}^{\mathrm{f}}$, and $\mathrm{n}_{\mathrm{m}}^{\mathrm{f}}$. We then determine the variables $\mathrm{f}_{\mathrm{s}}(\mathrm{p})$ and $f_{m}(p)$ for $p=1,2,3, \ldots$ by solving simultaneously eqs 18 and 19 , which lead to

$$
\begin{aligned}
& \text { In } n_{s}^{f} a^{3}+1-\frac{I_{B}}{2}\left[4 \pi I_{B}\left(s+m z^{2}+n_{s}^{f}+n_{m}^{f} z^{2}\right)\right]^{1 / 2}+ \\
& \frac{I_{B} p N\left(1-f_{s}(p)-f_{m}(p)\right)^{2}}{R^{2}(p)} \frac{\partial R}{\partial f_{s}}+\frac{2 I_{B} p N\left(1-f_{s}(p)-f_{m}(p)\right)}{R(p)}+ \\
& \quad 1.71 \frac{I_{B}}{a}-\frac{8 \pi R \gamma}{p N} \frac{\partial R}{\partial f_{s}}-\frac{4 \pi R^{2}}{p N} \frac{\partial \gamma}{\partial f_{s}}-\frac{3 R}{N^{2}} \frac{\partial R}{\partial f_{s}}+\frac{\pi^{2}}{3 R^{3}} \frac{\partial R}{\partial f_{s}}=0
\end{aligned}
$$

and

$$
\begin{gathered}
\text { In } n_{m}^{f} a^{3}+1-\frac{I_{B} z^{2}}{2}\left[\left.4 \pi\right|_{B}\left(s+m z^{2}+n_{s}^{f}+n_{m}^{f} z^{2}\right)\right]^{1 / 2}+ \\
\frac{I_{B} z p N\left(1-f_{s}(p)-f_{m}(p)\right)^{2}}{R^{2}(p)} \frac{\partial R}{\partial f_{m}}+ \\
\frac{\left.2 z\right|_{B} p N\left(1-f_{s}(p)-f_{m}(p)\right)}{R(p)}+1.71 \frac{I_{B}}{a} \frac{z(z+1)}{2}- \\
\frac{8 \pi z R \gamma}{p N} \frac{\partial R}{\partial f_{m}}-\frac{4 \pi z R^{2}}{p N} \frac{\partial \gamma}{\partial f_{m}}-\frac{3 z R}{N^{2}} \frac{\partial R}{\partial f_{m}}+\frac{z \pi^{2}}{3 R^{3}} \frac{\partial R}{\partial f_{m}}=0
\end{gathered}
$$


Finally, we obtain the concentration of p-aggregated polyelectrolyte chains, $\phi_{p}$, for $p=1,2,3, \ldots$ by substituting these values of $f_{s}(p)$ and $f_{m}(p)$ into eq $17 b$, in which $\mu_{\mathrm{p}}^{\circ}$ has the form of

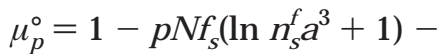

$$
\begin{aligned}
& \frac{p N f_{m}}{z}\left(\operatorname{ln~} n_{m}^{f} a^{3}+1\right)+\frac{I_{B} p^{2} N^{2}\left(1-f_{s}(p)-f_{m}(p)\right)^{2}}{R(p)}+ \\
& \frac{I_{B} p N}{2}\left(f_{s}+f_{m} z\right)\left[4 \pi I_{B}\left(s+m z^{2}+n_{s}^{f}+n_{m}^{f} z^{2}\right)\right]^{1 / 2}- \\
& 1.71 \frac{\mathrm{B}}{\mathrm{a}} \mathrm{pN}\left(\mathrm{f}_{\mathrm{s}}+\frac{\mathrm{f}_{\mathrm{m}}(\mathrm{z}+1)}{2}\right)+4 \pi \mathrm{R}^{2} \gamma+ \\
& \mathrm{p}\left(\frac{3}{2} \frac{\mathrm{R}^{2}}{\mathrm{Na}^{2}}+\frac{\pi^{2}}{6} \frac{\mathrm{Na}^{2}}{\mathrm{R}^{2}}-3.1449\right)
\end{aligned}
$$

Once $\phi_{p}, f_{s}(p)$, and $f_{m}(p)$ for $p=1,2,3, \ldots$ are obtained, the total concentration of polyelectrolytes $\phi$ in eq 1, the concentration of free negative monovalent ions $\mathrm{n}_{\mathrm{s}}^{\mathrm{f}}$ in eq 2, and the concentration of free negative multivalent ions $n_{m}^{f}$ in eq 3 are determined. These new values of $\phi$, $\mathrm{n}_{\mathrm{s}}^{\mathrm{f}}$ and $\mathrm{n}_{\mathrm{m}}^{\mathrm{f}}$ have to coincide with the initial values we input. Otherwise, we have to modify the initial conditions and repeat the numerical procedures described above. It should be noted that even for the polyel ectrolyte chains in the presence of only multivalent salt solutions, it is still possible for negative monovalent ions with initial concentration $\phi$ to condense to the chains.

The degree of polydispersity of the micelles is evaluated by computing the number-average $\mathrm{P}_{\mathrm{N}}$, the weightaverage $P_{W}$, and the $z$ average $P_{z}$, defined as

$$
\begin{aligned}
& P_{N}=M_{1} / M_{0} \\
& P_{W}=M_{2} / M_{1} \\
& P_{Z}=M_{3} / M_{2}
\end{aligned}
$$

where

$$
\mathrm{M}_{\mathrm{i}}=\sum_{\mathrm{p}=1,2, \ldots} \mathrm{n}_{\mathrm{p}} \mathrm{p}^{\mathrm{i}} \quad \mathrm{i}=1,2,3
$$

The moments $\mathrm{P}_{\mathrm{N}}$ and $\mathrm{P}_{\mathrm{W}}$ are accessible by osmotic and by light scattering measurements, respectively. The critical micelle concentration (cmc) is defined as the polyelectrolyte concentration at which

$$
\ln \mathrm{n}_{\mathrm{p}^{*}}=\ln \mathrm{n}_{1}
$$

where $n_{p^{*}}$ is the maximum of the number density of aggregates with $\mathrm{p}^{*}$ chains.

II.b. Stretched Single Polyelectrolyte Chains. The free energy functional per unit volume, $F N$, of polyelectrolyte chains with concentration $\phi$ in the extended state is constructed in the same way as that in the precipitated state, except that there is no interfacial free energy term $\mathrm{F}_{\text {en }} N$ for the extended single chains. That is

$$
\mathrm{F} N=\mathrm{F}_{\mathrm{tp}} N+\mathrm{F}_{\mathrm{id}} N+\mathrm{F}_{\mathrm{ti}} N+\mathrm{F}_{\mathrm{e}} N+\mathrm{F}_{\mathrm{es}} N+\mathrm{F}_{\mathrm{eb}} N
$$

where

$$
\begin{gathered}
\mathrm{F}_{\mathrm{tp}} N=\mathrm{k}_{\mathrm{B}} \mathrm{T} \frac{\phi}{\mathrm{N}} \ln \phi \mathrm{a}^{3} \\
\mathrm{~F}_{\mathrm{id}} N=\mathrm{k}_{\mathrm{B}} \mathrm{T} \frac{\phi}{\mathrm{N}}\left(\frac{3}{2} \frac{\mathrm{R}^{2}}{\mathrm{Na}^{2}}+\frac{\pi^{2}}{6} \frac{\mathrm{Na}}{\mathrm{R}^{2}}-3.1449\right)
\end{gathered}
$$

with $\mathrm{R}=\mathrm{Na} / 2$,

$$
\mathrm{F}_{\mathrm{eS}} \mathrm{N}=\mathrm{k}_{\mathrm{B}} \mathrm{T} \frac{\phi}{\mathrm{N}} \frac{\mathrm{I}_{\mathrm{B}}}{\mathrm{a}} \frac{1}{\mathrm{~N}} \mathrm{Z}_{\mathrm{eff}}^{2} \ln \mathrm{N}
$$

where the effective charge per chain $Z_{\text {eff }}$ is given in eq 14 setting $p=1$. Equation 29 is obtained from eq 13 setting $\mathrm{R}=\mathrm{Na}$ and adding the In $\mathrm{N}$ term, which is due to the anisotropy of the rods. ${ }^{14}$ At higher concentrations of $\phi, \mathrm{s}$, and/or $\mathrm{m}$, the effective charge of the stretched conformation is nearly zero. (Since in this regime the chains are self-avoi ding coils rather than stretched-like rods, our approach overestimates the entropy decrease of the stretched-coil conformation, which gives minor errors to the transition in this regime.) I on condensation leads to a further decrease in energy from the electrostatic short-range contacts between monomers and the condensed ions, which ignoring dipole-dipole interactions is given by ${ }^{22}$

$$
F_{e b} N=-k_{B} T \frac{\phi}{N} \frac{I_{B}}{a} \frac{N\left(f_{s}+f_{m} Z\right)}{2}
$$

The ideal translational energy term of free counterions $F_{t i}$ and the electrostatic interactions among all of the free ions $\mathrm{F}_{\mathrm{e}}$ are the same as those for precipitated chains and are given in eqs 10 and 11, respectively.

Both variables $f_{s}$ and $f_{m}$ are determined by equating the chemical potential of the free monovalent counterions and the condensed monovalent ions to the chains, which leads to

$$
\begin{array}{r}
\ln n_{s}^{f} a^{3}+1-\frac{I_{B}}{2}\left[\left.4 \pi\right|_{B}\left(s+m z^{2}+n_{s}^{f}+n_{m}^{f} z^{2}\right)\right]^{1 / 2}+ \\
\frac{2 I_{B}}{a}\left(1-f_{s}-f_{m}\right) \ln N+0.5 \frac{I_{B}}{a}=0
\end{array}
$$

and by equating the chemical potential of the free multivalent counterions and the condensed multivalent ions to the chains, which leads to

$$
\begin{aligned}
\operatorname{In} n_{m}^{f} a^{3}+ & 1-\frac{I_{B} z^{2}}{2}\left[\left.4 \pi\right|_{B}\left(s+m z^{2}+n_{s}^{f}+n_{m}^{f} z^{2}\right)\right]^{1 / 2}+ \\
& \frac{2 z I_{B}}{a}\left(1-f_{s}-f_{m}\right) \ln N+0.5 \frac{I_{B}}{a} z^{2}=0
\end{aligned}
$$

Once the variables of $f_{s}$ and $f_{m}$ are determined, the free energy of rodlike chains is calculated and compared with that of the precipitated chains.

\section{Results and Discussion}

The numerical results discussed here are for polyelectrolytes with $\mathrm{N}=500$ in the presence of multivalent salt with valence $z=2$ and $z=3$ solutions. In our calculations, we assume a very low concentration of monovalent salts (In sa $\left.{ }^{3}=-100\right)$, such that the amount of monovalent salt is ignored. The dimensionless Bjerrum length $\mathrm{I}_{\mathrm{B}} / \mathrm{a}$ is fixed to be equal to 2.8. 


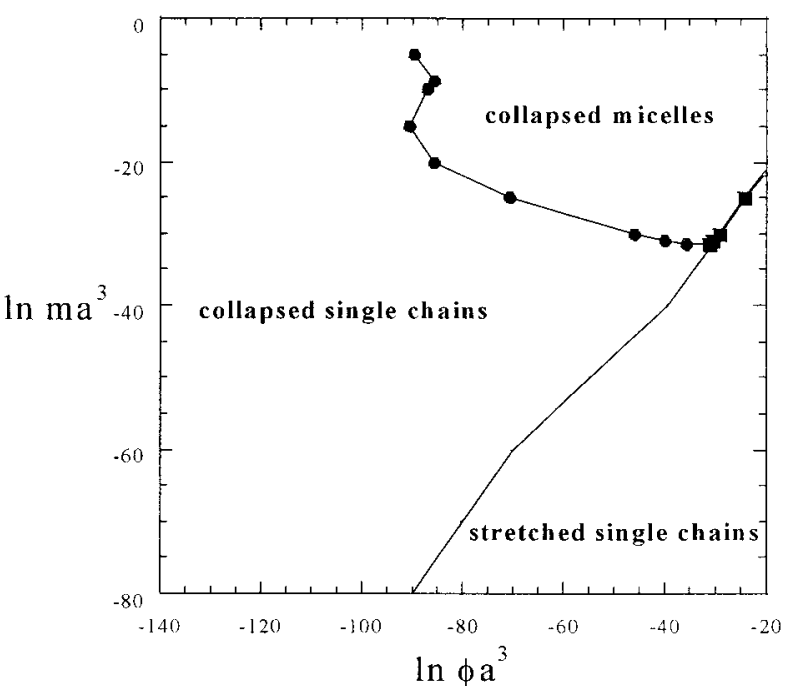

Figure 1. Numerical phase diagram in terms of logarithm of the concentration of multivalent salt ions (In $\mathrm{ma}^{3}$ ) and logarithm of the monomer concentration $\left(\operatorname{In} \phi \mathrm{a}^{3}\right)$ for dilute polyelectrolytes with $\mathrm{N}=500$ in the presence of divalent multivalent salt solutions. The logarithm of the concentration of monovalent salts In $\mathrm{sa}^{3}=-100$, and the dimensionless Bjerrum length $\mathrm{I}_{\mathrm{B}} / \mathrm{a}=2.8$.

In Figure 1, we present the numerical phase diagram for $\mathrm{z}=2$. The circles in Figure 1 correspond to the critical micelle concentrations (cmc) given by eq 25 , at which the chains start to form finite size micelles due to the significant amount of condensed negative multivalent ions. The squares, which denote the critical micelle dissolved concentration (cmdc), at which the formed $p$-aggregates start to dissolve, show a linear relationship $\phi \sim \mathrm{mz}$. This transition line from precipitated micelles to collapsed single chains al most overlaps with the transition line from collapsed single chains to nearly stretched rods, denoted as the sol id line in Figure 1. As expected, when the multivalent salt concentration $m$ is very low (In $m^{3} \leq-32$ ), only the transition from collapsed single chains to rodlike single chains is observed as the polyelectrolyte concentration increases to $\phi^{*} \cong \mathrm{mz}$. That is, when the total charges of the monomers are smaller than the charges of the multivalent salts, most of the monomer charges are compensated by the condensation of multivalent salt ions. The pol yel ectrolyte chains are collapsed due to the effective el ectrostatic attraction arising from these multivalent counterions condensed to the chains. In Figure $2 \mathrm{a}$, we plot the fraction of monomer charge from condensed multivalent and monovalent ions, $\mathrm{f}_{\mathrm{m}}$ and $\mathrm{f}_{\mathrm{s}}$, as a function of In $\phi \mathrm{a}^{3}$ when In $m \mathrm{a}^{3}=-40$. In Figure $2 \mathrm{~b}$ we plot the corresponding concentrations of free monovalent and multivalent ions, $\mathrm{n}_{\mathrm{s}}^{\mathrm{f}}$ and $\mathrm{n}_{\mathrm{m}}^{\mathrm{f}}$, with increasing $\ln \phi \mathrm{a}^{3}$. Clearly, when the chains are precipitated, $\mathrm{f}_{\mathrm{s}}=0$ and $f_{m} \rightarrow 1$. That is, none of the negative monovalent salt ions condense to the chains, and $n_{s}^{f}$ is equal to the initial monovalent ion concentration $\phi$. Though $\mathrm{f}_{\mathrm{m}} \rightarrow 1$ when $\phi<\phi^{*}$, the amount of negative multivalent ions condensed to the chains is not significant, so that $n_{m}^{f}$ still remains a constant. As $\phi$ increases such that $\phi \geq \phi^{*}, \mathrm{f}_{\mathrm{m}}$ drops dramatically to 0 , while $\mathrm{n}_{\mathrm{m}}^{\mathrm{f}}$ given in eq 3 reaches a minimum value and then increases to the initial concentration of multivalent salts. Though $f_{s}$ increases with increasing $\phi$, the number of free monovalent ions $n_{\mathrm{s}}^{\mathrm{f}}$ given in eq 2 still keeps a linear increasing relationship with $\phi$. Since there exist only a

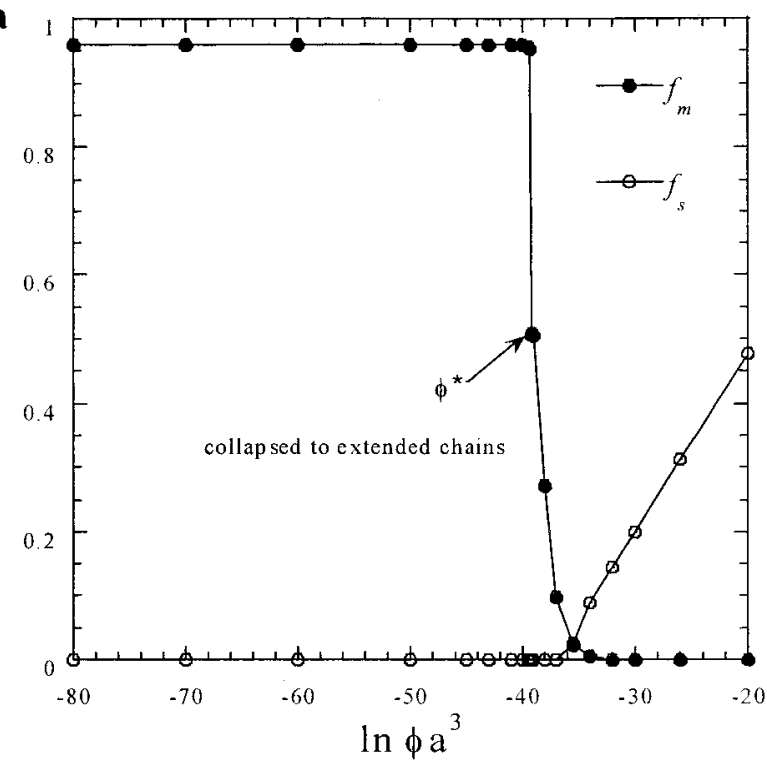

b

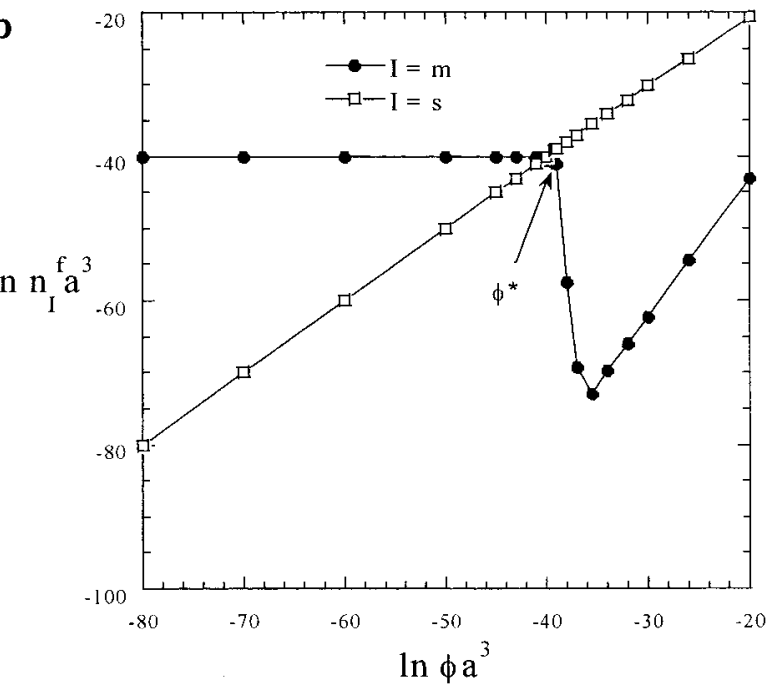

Figure 2. (a) Fraction of monomer charge compensated by condensed multivalent and monovalent ions, $f_{m}$ and $f_{s}$, vs In $\phi \mathrm{a}^{3}$, and (b) logarithm of the concentration of free multivalent and monovalent ions, $\ln \mathrm{n}_{\mathrm{m}}^{\mathrm{f}} \mathrm{a}^{3}$ and $\ln \mathrm{n}_{\mathrm{s}}^{\mathrm{f}} \mathrm{a}^{3}$, vs $\ln \phi \mathrm{a}^{3}$, for systems described in Figure 1 with In $\mathrm{ma}^{3} \stackrel{3}{=}-40$.

a few condensed monovalent ions along the chains, the electrostatic attraction energy is not strong enough to precipitate the chains. Therefore, the most stable state for the single chains is stretched when $\phi \geq \mathrm{mz}$. Notice that the favorable state is a stretched coil and not a stretched rod because at these concentrations there are enough free ions to screen the electrostatic interactions.

As the amount of multivalent salts increases such that $m \geq m * *\left(\ln m^{* *} a^{3}=-32\right)$, we find that the precipitated chains start to form micelles at a critical concentration $(\mathrm{cmc})$ lower than $\phi^{*} \cong \mathrm{mz}$. The micelles transform back to single collapsed chains and then to extended single chains with further increasing of $\phi$. The transition from collapsed to extended state still occurs at $\phi^{*} \cong \mathrm{mz}$. The transition from micelles to monomolecular aggregates (which occurs at about the cmdc value shown in Figure $3 a$ and defined below) is very close to the transition from collapsed to stretched chains (denoted by $\phi^{*}$ in Figure 3a). In Figure 3a, we plot the variation of the concentration of free chains $\left(\phi_{1}\right)$ as a 
$\mathbf{a}$
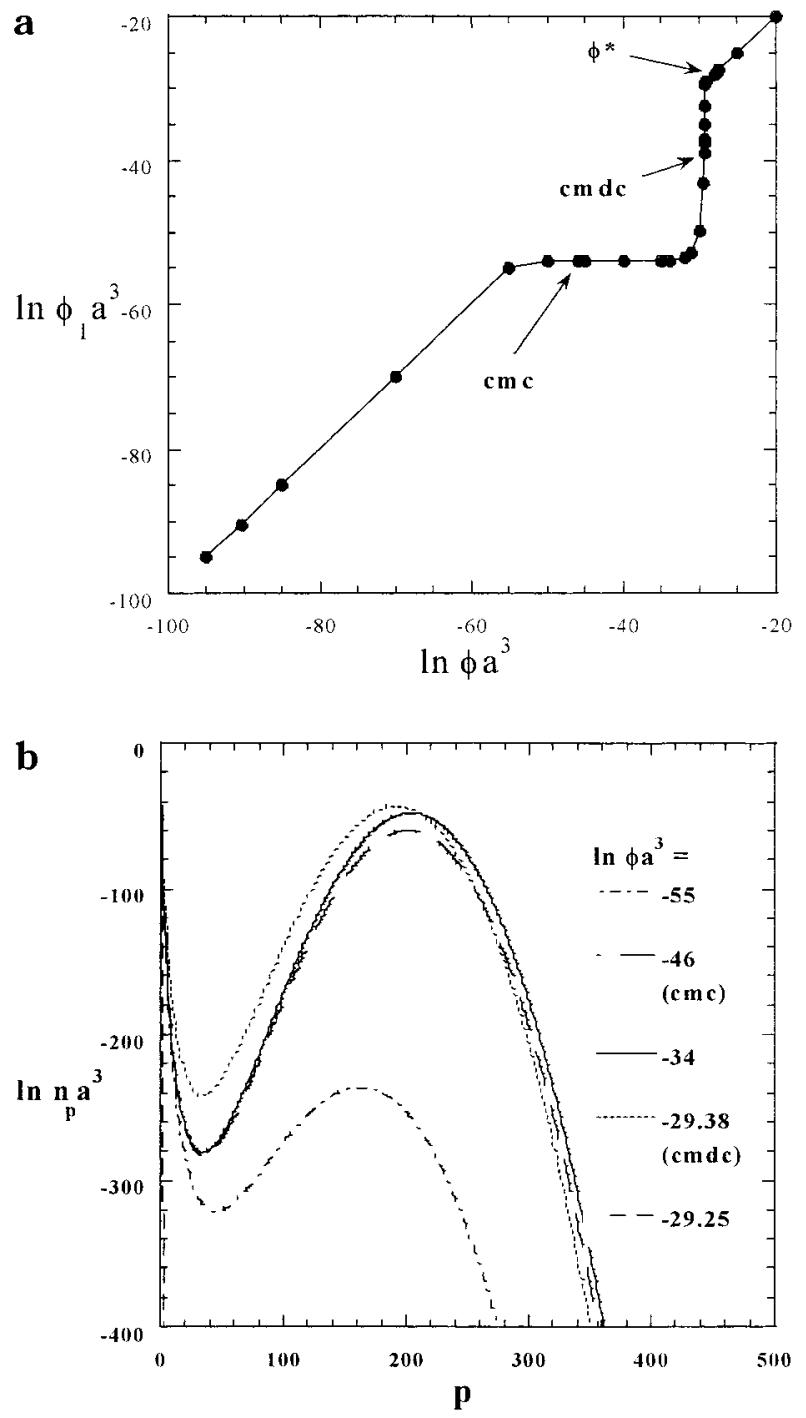
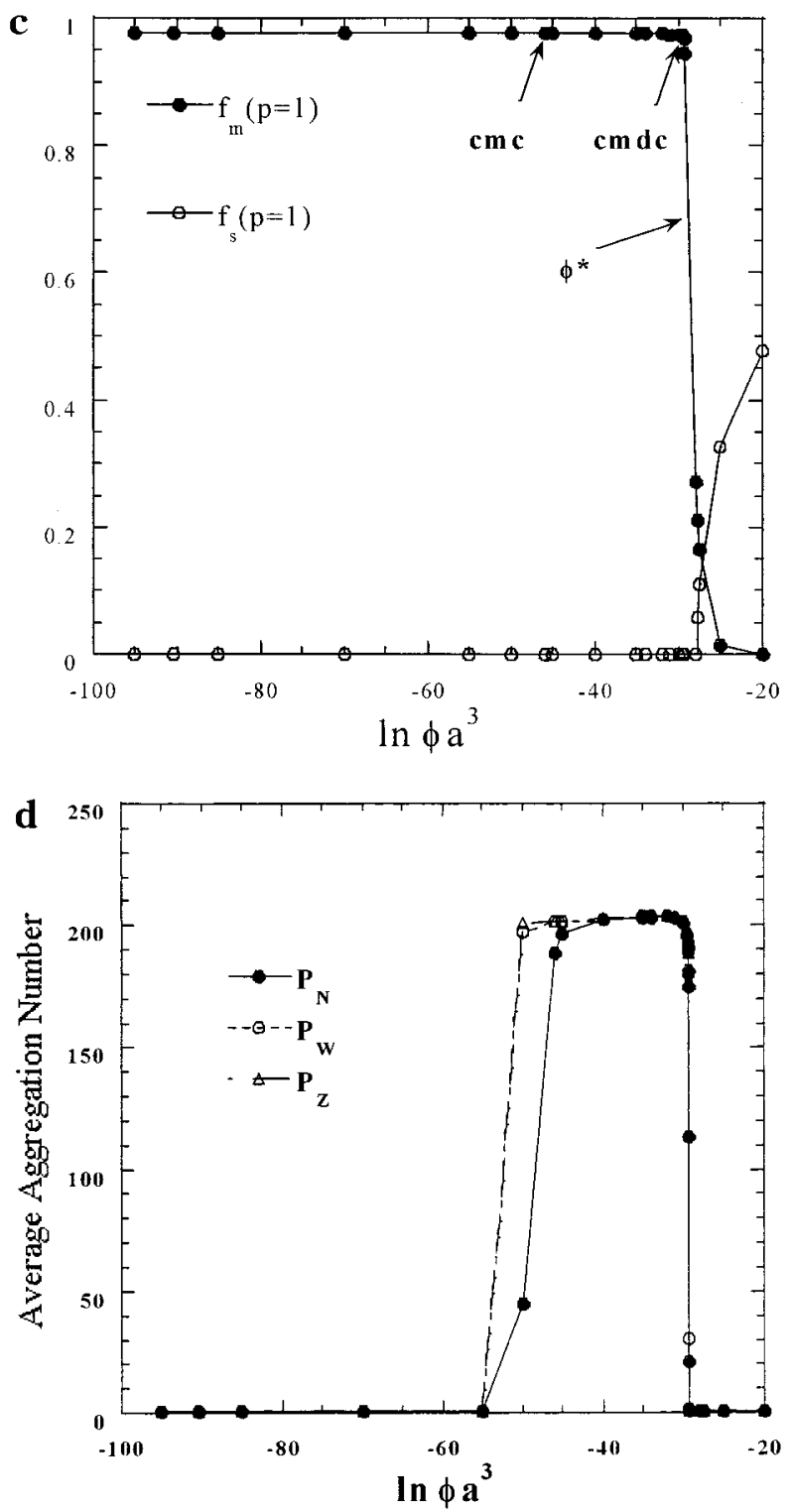

Figure 3. (a) Logarithm of the concentration of free chains $\ln \phi_{1} a^{3} v s \ln \phi a^{3}$, (b) logarithm of the number density of $p$-aggregated micelles (In $n_{p} a^{3}$ ) vs $p$ at various values of $\ln \phi a^{3}$, (c) variation of $f_{m}(p=1)$ and $f_{s}(p=1)$ as a function of In $\phi a^{3}$, and (d) average aggregation number vs In $\phi \mathrm{a}^{3}$, for systems described in Figure 1 with In $\mathrm{ma}^{3}=-30$.

function of the total concentration of polyelectrolytes $(\phi)$ with In $\mathrm{ma}^{3}=-30$. In Figure $3 b$, we also show the typical results of number density of $p$-aggregates $\ln n_{p} a^{3}$ vs $p$ at various values of polyelectrolyte concentration $\phi$ corresponding to various states of the chains. As shown in Figure 3a, when the polyelectrolyte concentration is well bel ow $\mathrm{cmc}$ ( $\left.\ln \phi \mathrm{a}^{3}=-46\right)$, the precipitated chains remain single since $\phi_{1} \sim \phi$. As $\phi$ increases from $\mathrm{cmc}$, the concentration of free chains remains a constant. This manifests the fact that there are more aggregates with $p^{*}>1$ chains with increasing $\phi$, until when In $\phi \mathrm{a}^{3}$ is cl ose to a value of -29.38 , the free chain concentration increases abruptly, and the number density of p-aggregated micelles decreases. In particular, when In $\phi \mathrm{a}^{3}=-29.38$, we observe that though there exists a local maximum of In $n_{p} a^{3}$ at $p=p^{*}, \ln n_{p^{*}}=\ln$ $\mathrm{n}_{1}$, as shown in Figure $3 \mathrm{~b}$. We define the concentration at which $\ln n_{p^{*}}=\ln n_{1}$, as the critical micelle dissolved concentration (cmdc). As $\phi$ increases above the cmdc such as when $\ln \phi \mathrm{a}^{3}=-29.25$, the micelles dissolve, and these polyelectrolyte chains become single monomolecular aggregates again, as shown in Figure $3 b$.
Furthermore, we find that the value of cmdc is very cl ose to the concentration at which the collapsed single chains transform to the stretched rods, denoted as $\phi^{*}$. Once the concentration is above $\phi^{*}$, these single chains become stretched. Similar to the case for very low concentration of multivalent salts, we observe that no matter when the precipitated chains are single or micelles, each chain is almost neutral by the condensation of multivalent salt counterions. This is shown in Figure $3 c$ where we plot $f_{m}(p=1)$ and $f_{s}(p=1)$ as a function of In $\phi \mathrm{a}^{3}$ with $\ln \mathrm{ma}^{3}=-30$. As $\phi$ increases to the stretched-like state, $f_{m}$ decreases dramatically to 0 ; however, the charge of monomers is still partly compensated by the condensation of monovalent counterions. With the polyelectrolyte chains aggregating to form the micelles, $f_{s}(p)$ remains 0 , while $f_{m}(p)$ increases and approaches 1 as $p$ increases. In Figure $3 d$ we plot the average aggregation numbers defined in eq 23 as a function of polyelectrolyte concentration $\phi$. As can be seen clearly, the formed p-aggregated micelles are finite size with the typical value of average aggregation number equal to 205. 

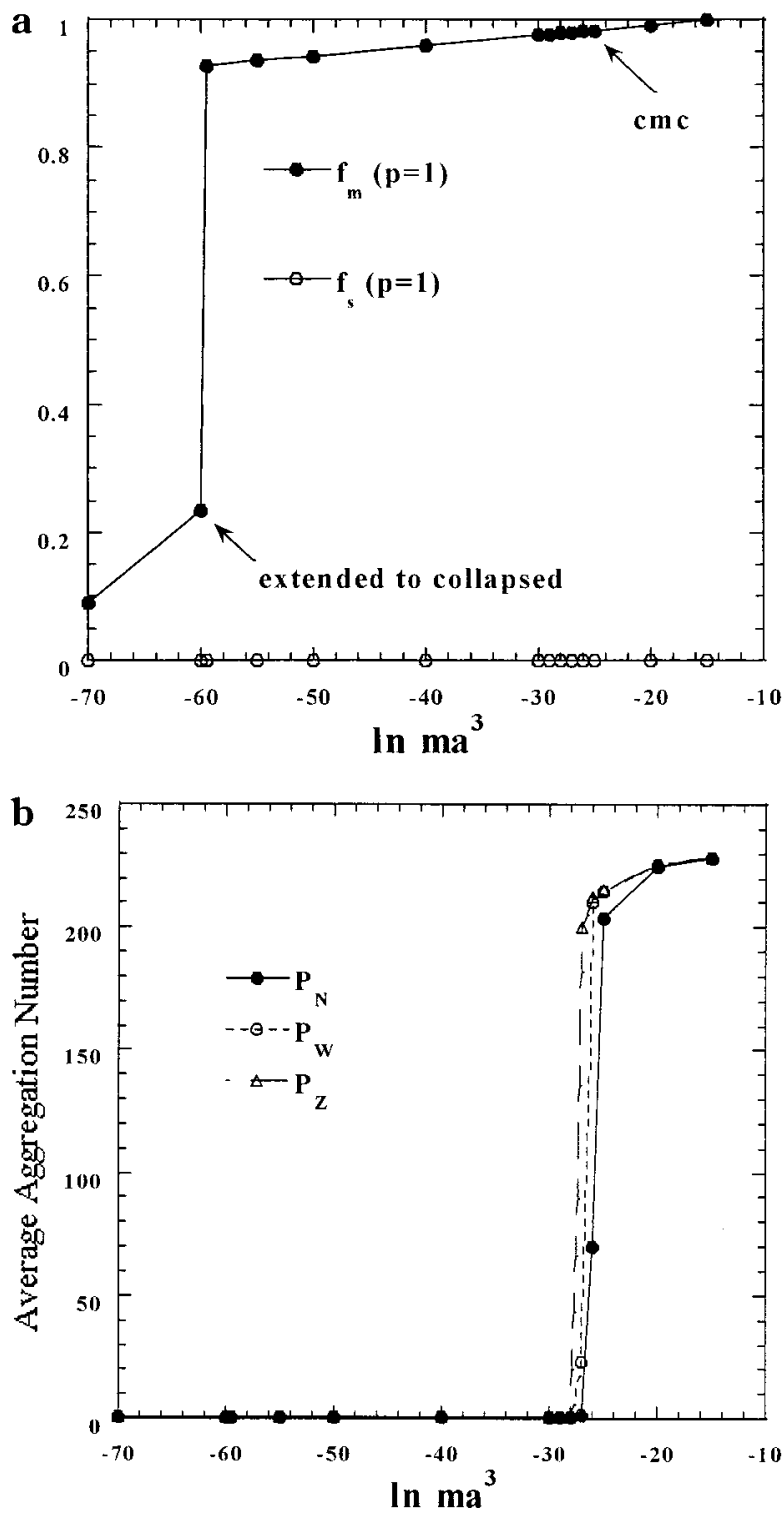

Figure 4. (a) Variation of $f_{m}(p=1)$ and $f_{s}(p=1)$ as a function of logarithm of the concentration of multivalent salt counterions (In $\left.\mathrm{ma}^{3}\right)$, and (b) average aggregation number vs In $\mathrm{ma}^{3}$, for systems described in Figure 1 with In $\phi \mathrm{a}^{3}=-70$.

The multimolecular aggregation behavior, which occurs when the multivalent salt concentration is above a critical value $\mathrm{m}^{* *}$, is driven by a sufficient amount of condensed multivalent salt counterions. These condensed counterions act as a bad solvent to the chains given that they generate effective attractions between the charged monomers. As shown in Figure 4a, where we plot $f_{m}(p=1)$ as a function of multivalent salt concentration at a fixed value of In $\phi a^{3}=-70, f_{m}(p=1)$ increases as $m$ increases. That is, with more multivalent salts added to the solutions, more multivalent ions condense to the polyelectrolyte chains. These counterion-mediated attractions drive more chains to aggregate into micelles, as shown in Figure $4 \mathrm{~b}$ where we plot the average aggregation numbers as a function of In $\mathrm{ma}^{3}$.

When $m$ is very large, such as when In $\mathrm{ma}^{3}=-8.7$, we only observe the transition from single precipitated chains to finite size aggregates with increasing $\phi$. For example, in parts a and b of Figure 5, we plot In $\phi_{1} a^{3}$ vs In $\phi a^{3}$ and In $n_{p} a^{3}$ vs $p$ as a function of In $\phi \mathrm{a}^{3}$, respectively. Once the precipitated single chains form the micelles when $\phi \geq \mathrm{cmc}$, $\phi_{1}$ remains almost a $\mathbf{a}$

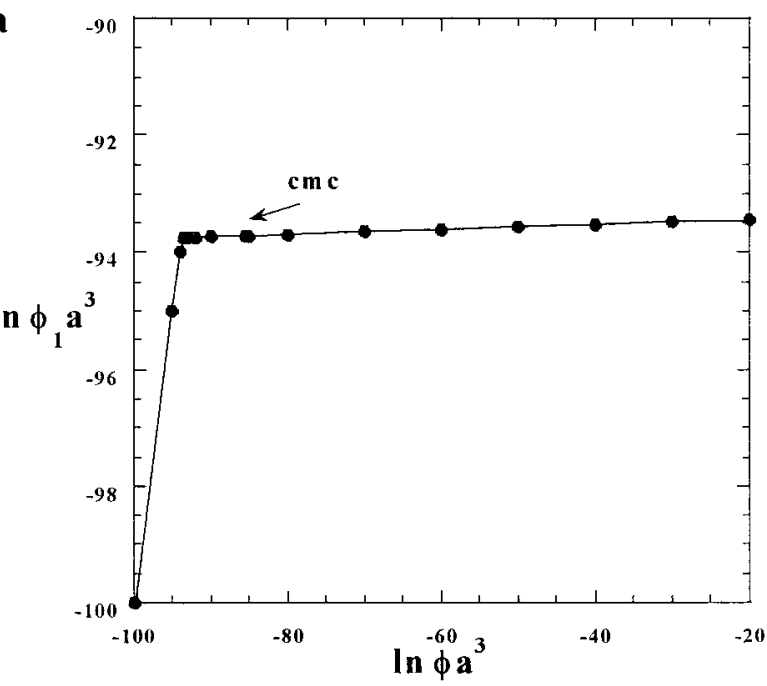

b
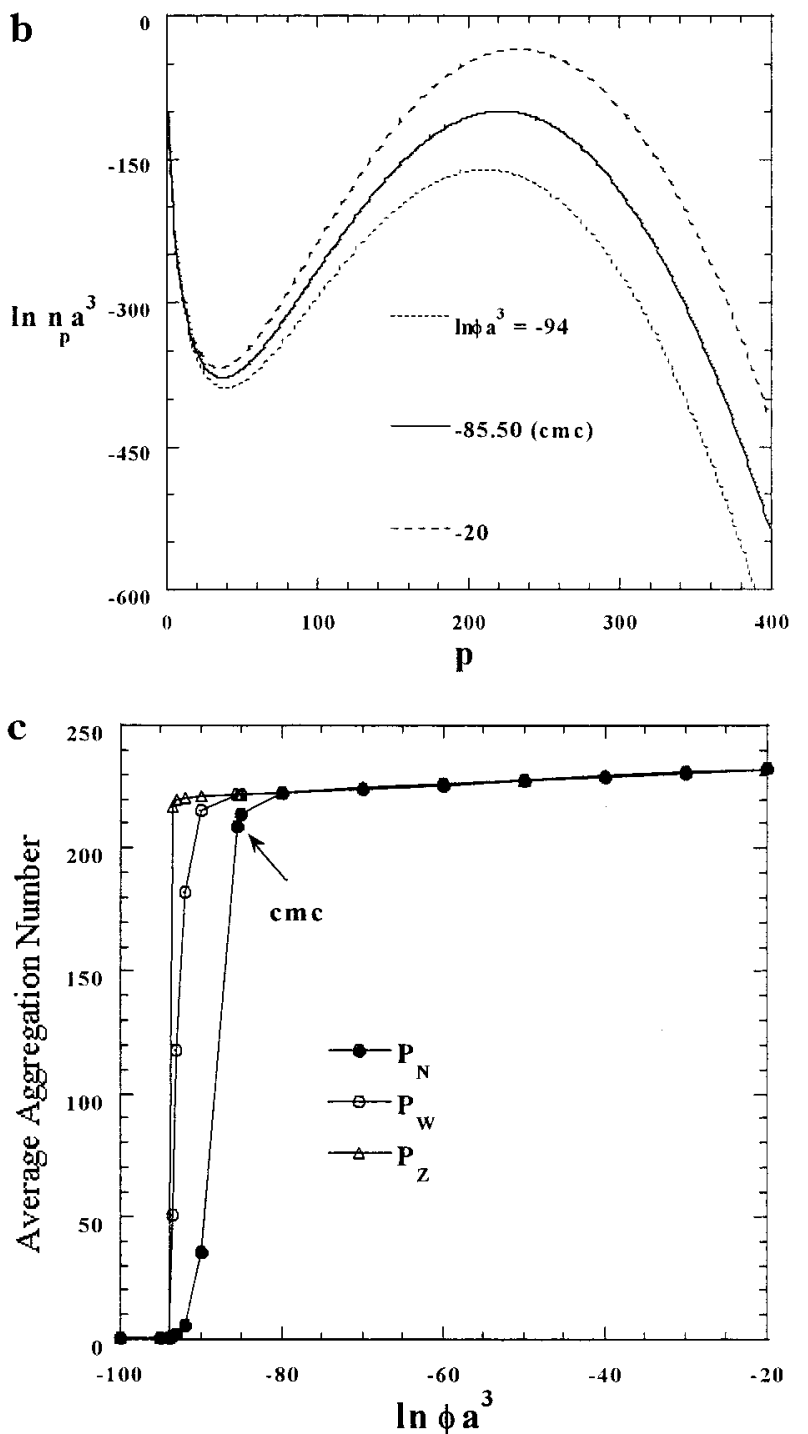

Figure 5. (a) Logarithm of the concentration of free chains In $\phi_{1} a^{3}$ vs In $\phi a^{3}$, (b) logarithm of the number density of p-aggregated micelles ( $\left.\ln n_{p} a^{3}\right)$ vs $p$ at various values of $\ln \phi a^{3}$, and (c) average aggregation number vs In $\phi \mathrm{a}^{3}$, for systems described in Figure 1 with $\ln \mathrm{ma}^{3}=-8.7$.

constant with increasing $\phi$, which manifests the fact that the amount of the formed p-aggregates increases as $\phi$ increases, as shown in Figure 5b. Since the initial 
multivalent salt concentration is very high, we observe that no matter whether the chains are free or aggregated, none of the monovalent counterions condense to the chains. However, the preci pitated chains are neutral with the condensation of multival ent ions. It should be noticed that even when In $\phi \mathrm{a}^{3}$ increases to -20 , due to the effects of sufficient condensed multivalent counterions, these precipitated chains still form very monodisperse micelles, as shown in Figure $5 c$, where we plot the average aggregation numbers as a function of polyelectrolyte concentration.

Analytical results of finite-size aggregation behavior of polyelectrolytes can be obtained by minimizing $\left(\mu_{\mathrm{p}}^{\circ}-\mathrm{p} \mu_{1}^{\circ}\right) / \mathrm{p}$ in eq $17 \mathrm{~b}$ with respect to $\mathrm{p}$, i.e.,

$$
\frac{\partial\left[\left(\mu_{\mathrm{p}}^{\circ}-\mathrm{p} \mu_{1}^{\circ}\right) / \mathrm{p}\right]}{\partial \mathrm{p}}=0 \text { at } \mathrm{p}=\mathrm{p}^{*}
$$

The scaling results of $p^{*}$ are easily obtained by inserting $\mu_{\mathrm{p}}^{\circ}$ in eq 22 and the values of $\mathrm{f}_{\mathrm{s}}\left(\mathrm{p}^{*}\right)=0$ and $\mathrm{f}_{\mathrm{m}}\left(\mathrm{p}^{*}\right)=1$ into eq 33,

$$
\begin{aligned}
-\left(p^{*}\right)^{-4 / 3} N^{2 / 3} \frac{I_{B}}{a} z\left(1+\frac{1}{z}\right)^{-1 / 3}+ & \\
& \left(p^{*}\right)^{-1 / 3} N^{-1 / 3}\left(1+\frac{1}{z}\right)^{2 / 3}=0
\end{aligned}
$$

from which we obtain

$$
p^{*} \sim N z \frac{I_{B}}{a}
$$

Notice that the first term and the second term on the left-hand side of eq 34 is contributed from the el ectrostatic surface energy in eq 16 and the first term on the right-hand side of the entropy term in eq 8 . Without the nonlinear additive in $p$ term via the $R(p)=(p N)^{1 / 3}$ a in the chain entropy term added to the free energy, the only possible value of $p^{*}$ from eq 33 is $\infty$.

With increasing the valence of multival ent salts, due to the stronger el ectrostatic attraction free energy generated from the condensed multivalent salt counterions, we expect that the finite size multimolecular aggregation behavior occurs at a lower critical salt concentration $\mathrm{m}^{* *}$. For example, in Figure 6 we show the numerical phase diagram for $z=3$. In comparison with the results for $z=2$, similar phase behavior is observed; however, the region of micelles is much enlarged. Notice that though $z=2$ is a condensing agent in poly(acrylic acid) $S^{5}$ it is only a marginal condensing ion for PSS and DNA. We therefore conclude that our equation overestimates the decrease of the electrostatic energy of the precipitated state (and also overestimates the chain entropy reduction of the chains in the stretched-coil state).

\section{Conclusions}

We devel op a simple thermodynamic model for polydisperse micellar suspensions, which includes both monovalent and multivalent ion condensations along the chains. We determine the number density of aggregates with $p$ chains $\left(n_{p}\right)$ by equating the chemical potential of all the p-aggregated chains with $p=1,2,3, \ldots$, and equating the chemical potential of the condensed counterions in the aggregates with $p=1,2,3, \ldots$ and

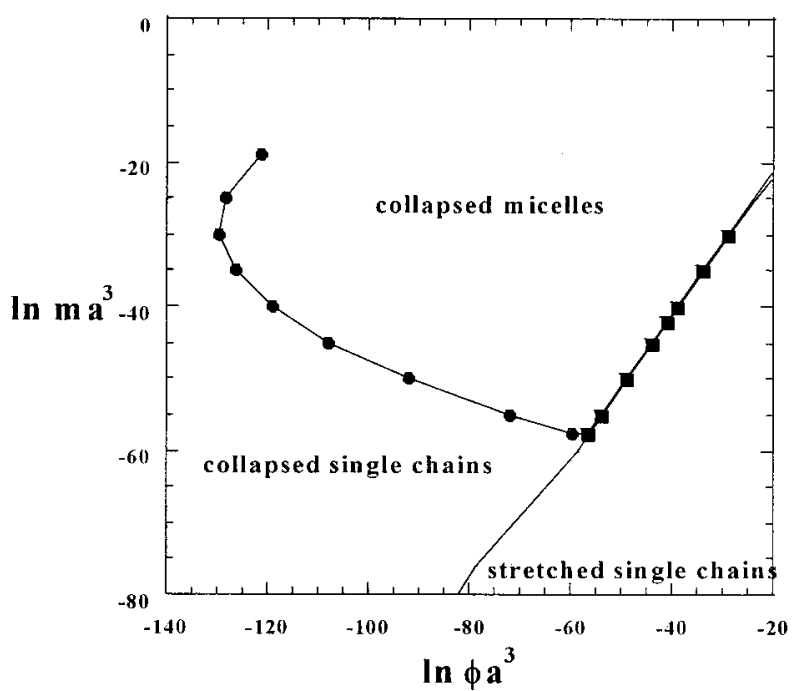

Figure 6. Numerical phase diagram in terms of logarithm of the concentration of multivalent salt ions (In $\mathrm{ma}^{3}$ ) and logarithm of the monomer concentration (In $\left.\phi \mathrm{a}^{3}\right)$ for dilute polyelectrolytes with $\mathrm{N}=500$ in the presence of trivalent multivalent salt solutions. The logarithm of the concentration of monovalent salts In $\mathrm{sa}^{3}=-100$, and the dimensionless Bjerrum length $\mathrm{I}_{\mathrm{B}} / \mathrm{a}=2.8$.

the free counterions. We calculate the free energy of the stretched chains and compare it with the free energy of the precipitated chains.

Finite size micelles are predicted in dilute polyelectrolyte solutions in the presence of multivalent salts with valence $z$ only if a nonlinear additive term in the number of chains $p$ is used in the entropy decrease of the aggregated chains. This situation arises only in chains constrained to stretched conformations, such as semiflexible chains growing from a toroid seed formed during the monomolecular collapse of long chains at low concentrations of chains. Flexible unconstrained chains, however, lead to aggregates with an infinite number of chains. When the concentration of multivalent salts $m$ is low, the monomolecular collapsed chains transform to nearly stretched chains, increasing the monomer concentration above $\phi^{*} \cong \mathrm{mz}$. When $\phi<\phi^{*}$, the chains are nearly neutralized by condensed multivalent counterions. Though there are condensed counterions along the chains when the $\phi \geq \phi^{*}$, the amount is not large enough to drive the precipitation of the chains. At larger concentrations of multivalent salts $\left(m>m^{* *}\right)$ the monomolecular collapsed chains form multimolecular aggregates upon increasing $\phi$. If the aggregates of collapsed chains are assumed to be monodisperse and homogeneous (i.e., containing $p^{*}$ chains in a sphere of $\left.R(p)=(p N)^{1 / 3} a\right)$, the minimization of free energy functional yields $\mathrm{p}^{*} \sim \mathrm{Nzl}_{\mathrm{B}} / \mathrm{a}$. With increasing the valence of multivalent salts, the finite size multimolecular aggregation behavior occurs at a lower critical salt concentration $\mathrm{m}^{* *}$ since the electrostatic attractions increase with increasing $z$.

At constant $\mathrm{m}>\mathrm{m}^{* *}$, the micelles redissolve with further increasing $\phi$, at about $\phi^{*}$, where $\phi^{*} \sim \mathrm{mz}$ is the concentration at which the chains transform from collapsed to stretched. The approximations used here are not good enough to determine whether the micelles redissolve directly into stretched chains by increasing $\phi$ or whether they transform into monomolecular collapsed chains and then into stretched chains. Since as $\mathrm{m}$ increases also $\phi^{*}$ increases, the screening of the 
electrostatic interactions may lead to necklaces 44,45 and/ or stretched-coil statistics at the redissolution, leading to lower free energies than the stretched state used here. Moreover, for large $m$ values, the transition at $\phi^{*}$ may occur at concentrations where there is sufficient overlapping between the chains, which is ignored here. Finally, at large $m$ values the continuum Debye-Huckel free energy used for the free counterions breaks down due to Bjerrum association of the free multivalent and monovalent salt ions.

Acknowledgment. Ching-I Huang acknowledges the financial support from the National Science Council of the Republic of China through Grant NSC 89-2216E-011-043. Monica Olvera de la Cruz acknowl edges the support of the National Institutes of Health (Grant GM62109).

\section{References and Notes}

(1) Widom, J .; Baldwin, R. J . J . Mol. Biol. 1980, 144, 431

(2) Narh, K. A.; Keller, A. J. Polym. Sci., Part B: Polym. Phys. 1993, 31, 231.

(3) Yoshikawa, K.; Takahashi, M.; Vasilevskaya, V. V.; Khokhlov, A. R. Phys. Rev. Lett. 1996, 76, 3029.

(4) Raspaud, E.; Olvera de la Cruz, M.; Sikorav, J . L.; Livolant, F. Biophys. J. 1998, 74, 381.

(5) Sabbagh, I.; Delsanti, M.; Lesieur, P. Eur. Phys. J . B 1999, $12,253$.

(6) Stevens, M.; Kremer, K. J . Chem. Phys. 1995, 103, 1669.

(7) Lyubratsev, A. P.; Nordenskild, L. J . Phys. Chem. 1997, 101, 4335.

(8) Gronbech-J ensen, N.; Mashl, R.J .; Bruinsama, R. F.; Gel bart W. M. Phys. Rev. Lett. 1997, 78, 2477.

(9) Arenzon, J. J .; Stilck, J . F.; Levin, Y. Eur. Phys. J . B 1999, $12,79$.

(10) Lee, N.; Thirumalai, D. http://xxx.Ian1.gov/abs/cond-mat/ 9907199. Lee, N.; Thirumalai, D. J . Chem. Phys. 2000, 113, 5126.

(11) Stevens, M. Biophys. J . 2001, 80, 130.

(12) Olvera de la Cruz, M.; Belloni, L.; Delsanti, M.; Dalbiez, J. P.; Spalla, O.; Drifford, M. J . Chem. Phys. 1995, 103, 5781.

(13) Raspaud, E.; Chaperon, I.; Leforestier, A.; Livolant, F. Biophys. J. 1999, 77, 1547.

(14) Gonzalez-M ozuelos, P.; Olvera de la Cruz, M.J . Chem. Phys. 1995, 103, 3145.

(15) Wittner, J .; J ohner, A.; J oanny, J . F. J . Phys., II 1995, 5, 635.

(16) Barrat, J .; J oanny, J . F. Adv. Chem. Phys. 1996, 94, 1. Ha, B. Y.; Liu, A. J . Phys. Rev. Lett. 1997, 79, 1289. Ray, J .; Manning, G. S. Macromolecules 2000, 33, 2901.

(17) Rouzina, I.; Bloomfield, V. A. J . Phys. Chem. 1996, 100, 9977.

(18) Solis, F.J .; Olvera de la Cruz, M. Phys. Rev. E 1999, 60, 4496.

(19) Schiessel, H.; Pincus, P. Macromol ecules 1988, 21, 7953.

(20) Brilliantov, N. V.; Kuznetsov, D. V.; Klein, R. Phys. Rev. Lett. 1998, 81, 1433.

(21) Solis, F. J .; Olvera de la Cruz, M. J . Chem. Phys. 2000, 112, 2030.

(22) Solis, F. J .; Olvera de la Cruz, M. Eur. Phys. J . E 2001, 4 143. Solis, F. J .; Olvera de la Cruz, M. Eur. Phys. Direct E 2000, 1,1 .

(23) Muthukumar, M.; Carri, G. J . Chem. Phys. 1999, 111, 1765.
(24) Vasilevskaya, V. V.; Khokhlov, A. R.; Kidoaki, S.; Yoshikawa, K. Biopolymers. 1997, 41, 51.

(25) Manning, G. S. J . Chem. Phys. 1969, 51, 924. Oosawa, F. Polyelectrolytes; Marcel Dekker: New York, 1971.

(26) Alexander, M.; Chaikin, P. M.; Grant, P.; Morales, G. J .; Pincus, P.; Hone, D. J . Chem. Phys. 1984, 80, 5776. Belloni, L. Ph.D. Thesis, Paris, France, 1982.

(27) Fixman, M.J. . Chem. Phys. 1979, 70, 4995.

(28) Wigner, E. P. Trans. Faraday Soc. 1938, 34, 678

(29) Matulis, D.; Rouzina, I.; Bloomfield, V. A. J . Mol. Biol. 2000 296, 1053. Koltover, I.; Wagner, K.; Safinya, C. R. Proc. Natl. Acad. Sci. 2000, 97, 14046.

(30) Kjellander, R.; Akesson, T.; I onsson, B.; Marcelja, S. J . Chem. Phys. 1992, 97, 1424.

(31) An elementary introduction to the Madelung constant appears in section II.8.3 of: Feynman, R. P.; Leighton, R. B.; Sand, M. The Feynman Lectures in Physics; AddisonWesley: Reading, MA, 1964.

(32) Ha, B. Y.; Liu, A. J. Phys. Rev. Lett. 1998, 81, 1011.

(33) Bloomfield, V. A. Polymer and Polyelectrolyte Behavior in DNA. In Nucleic Acids: Structures, Properties and Functions; University Science Books: Mill Valley, 1997.

(34) Park S. Y.; Harries, D.; Gel bart, W. M. Biophys. J . 1998, 75 714.

(35) Ha, B. Y.; Liu A. J . Europhys. Lett. 1999, 46, 624.

(36) Stevens, M. Phys. Rev. Lett. 1999, 82, 101.

(37) Borue, V. Y.; Erukhimovich, Y. I. Macromolecules 1988, 21 3240.

(38) Mahdi, K. A.; Olvera de la Cruz, M. Macromolecules 2000 $33,7649$.

(39) Huang, C.; Olvera de la Cruz, M.; Delsanti, M.; Guenoun, P Macromol ecules 1997, 25, 88019.

(40) J ohnson, R. A.; Nagarajan, R. Colloids Surf. A 2000, 167, 21.

(41) Grosberg, A. Y. Biofizika (URSS) 1979, 24, 32. Grosberg, A Y.; Erukhimovich, I. Y.; Shakhnovich, E. I. Biopolymers 1982 $21,2413$.

(42) de Gennes, P. G.; Pincus, P.; Velasco, R. M.; Brochard, F. J Phys. (Paris) 1976, 37, 1461.

(43) Leibler, L.; Orland, H.; Wheeler, J . C. J . Chem. Phys. 1983 79,3550

(44) Dobrynin, A. V.; Rubinstein, M.; Obukhov, S. P. Macromolecules 1996, 29, 2974. Dobrynin, A. V.; Rubinstein, M. Macromolecules 2000, 34, 1964.

(45) Solis, F. J .; Olvera de la Cruz, M. Macromol ecules 1998, 31 , 5502. Pickett, G. T.; Balazs, A. C. Langmuir 2001, 17, 5111.

(46) Micka, U.; Kremer, K. Europhys. Lett. 2000, 49, 189.

(47) Waigh, T. A.; Ober, R.; Williams, C. E.; Galin, J . C. Macromolecules 2001, 34, 1973.

(48) Levin, Y. Physica A 1999, 265, 432

(49) Solis, F.J .; Olvera dela Cruz, M. http://xxx.Ian1.gov/abs/condmat/0010065.

(50) Conway, B. E.; Bockris, J . O'M.; Yeager, E. Comprehensive Treatise of Electrochemistry; Plenum Press: New York, 1983 Vol. 5.

(51) McQuarrie, D. A. Statistical Mechanics; Harper and Row: New York, 1976

(52) Varela, L. M.; Perez-Rodriguez, M.; Garcia, M.; Sarmiento, F.; Mosquera, V. J . Chem. Phys. 1998, 109, 1930.

(53) Gonzalez-Mozuel os, P.; Bagatella-Flores, N. Physica A 2000 286, 56.

(54) Mahdi, K. A.; Olvera de la Cruz, M. Macromolecules 2000, 33, 7649.

(55) Campus, M.; Solis, F. J .; Olvera de la Cruz, M., unpublished. MA010717M 